Research Article

\title{
Extra Pulmonary Tuberculosis (EPTB): A Diagnostic Enigma and how to Improve Detection?
}

\author{
Sanjeev H Bhat', $\underline{\text { SP Burma }}^{2}$ \\ ${ }^{1}$ Associate Professor, Department of Microbiology, ANIIMS, Port Blair. \\ ${ }^{2}$ Senior Specialist, Department of Chest \& TB, GB Pant Hospital, Port Blair. \\ DOI: https://doi.org/10.24321/2454.8642.201906
}

\section{I $\quad \mathbf{N} \quad \mathbf{F}$}

\section{Corresponding Author:}

SP Burma, Department of Chest \& TB, GB Pant Hospital, Port Blair.

E-mail Id:

microaniimspb@gmail.com

Orcid Id:

https://orcid.org/0000-0001-5424-0673

How to cite this article:

Bhat SH, Burma SP. Extra Pulmonary Tuberculosis (EPTB): A Diagnostic Enigma and how to Improve Detection? Rec Adv Path Lab Med 2019; 5(2): 8-10.

Date of Submission: 2019-07-05

Date of Acceptance: 2019-08-22

\section{$\begin{array}{llllllll}\mathbf{A} & \mathbf{B} & \mathbf{S} & \mathbf{T} & \mathbf{R} & \mathbf{A} & \mathbf{C} & \mathbf{T}\end{array}$}

Tuberculosis (TB), for many centuries, has been the most important of human infections in its global prevalence, devastating morbidity and massive mortality. The term EPTB has been used to describe isolated occurence of TB at body sites other than the lungs. The most common anatomic sites affected by extrapulmonary TB are lymph nodes, pleura, bones \& joints.

Material \& Method: The SOP in our laboratory was to screen any sterile body fluid and exudate specimen received for AFB, irrespective of requisition.

Result: During the study period, a total of 1572 sterile body fluid and exudate specimen were received at the Clinical Microbiology Laboratory for bacterial culture \& sensitivity. Among the 1572 specimen screened, 06 specimens were positive for AFB on smear microscopy.

Conclusion: Our findings are based on only by introducing an additional investigation of low sensitivity, i: e smear microscopy. The proportion of cases detected could be higher if Mycobacterial culture or a Rapid molecular method was performed on the specimens.

Keywords: Tuberculosis, Extra Pulmonary Tuberculosis, AFB

\section{Introduction}

Tuberculosis (TB), for many centuries, has been the most important of human infections in its global prevalence, devastating morbidity and massive mortality. ${ }^{1}$ The World Health Organization (WHO) estimated 10.4 million incident cases of TB and 1.67 million TB deaths in 2017. ${ }^{2}$ Tuberculosis is a multisystem disease with myriad presentations and manifestations; it can affect any organ or tissue, excluding only the hair and nails. Effective control programmes are necessary for controlling the spread of the disease. ${ }^{3}$ Poverty, overcrowding, poor infection control practices and lack of financial and technical resources have hindered the effective implementation and the success of the control programmes. ${ }^{4}$

The term EPTB has been used to describe isolated occurence of TB at body sites other than the lungs. ${ }^{5}$ The most common anatomic sites affected by extrapulmonary TB are lymph nodes, pleura, bones \& joints, urogenital tract and meninges ${ }^{6}$. However data on extra pulmonary tuberculosis is limited. This is in part due to lack of awareness and clinical 
suspicion. Secondly, there is under estimation and under reporting of EPTB either due to lack of awareness or to non-availability of reliable diagnostic method.

The study was undertaken to explore the feasibility and utility of introducing an additional investigation which would aid in detection of EPTB.

\section{Material and Methods}

The study was performed in the department of Clinical Microbiology, of medical college teaching Hospital. The SOP in our laboratory was to screen any sterile body fluid and exudate specimen received for $A F B$, irrespective of requisition. A thorough review was made of available records from 2015 to 2018. All above such sample are stained by conventional Ziehl-Neelsen staining and observed for Mycobacteria. Further the sample was centrifuged at $6000 \mathrm{rpm}$ for 15 minutes and a smear, made from the deposit, was stained for AFB. Presence of two or more AFB was considered significant and the preliminary report was communicated to the treating doctor for clinical correlation with a request for repeat specimen, wherever possible. The specimen was also screened by CB NAAT for rapid detection of Mycobacterium tuberculosis.

\section{Exclusion Criteria}

Body fluid such as bile was not included in the study. Exudate sample received as swabs were not considered as they were unsuitable for demonstration of AFB.

\section{Result}

During the study period, a total of 1572 sterile body fluid and exudate specimen were received at the Clinical Microbiology Laboratory for bacterial culture \& sensitivity.

Table I.Distribution of specimen

\begin{tabular}{|c|c|c|}
\hline S. No. & Sample & Total (\%) \\
\hline 1. & Pus & $588(37.40)$ \\
\hline 2. & CSF & $220(13.99)$ \\
\hline 3. & Pleural & $172(10.94)$ \\
\hline 4. & Ascetic fluid & $165(10.50)$ \\
\hline 5. & Synovial fluid & $87(5.53)$ \\
\hline 6. & Endotracheal aspirate & $39(2.49)$ \\
\hline 7. & Peritoneal & $34(2.16)$ \\
\hline 8. & Endometrium & $29(1.84)$ \\
\hline 9. & Tissue & $26(1.65)$ \\
\hline 10. & Gastric aspirate & $12(0.76)$ \\
\hline 11. & Others & $200(12.72)$ \\
\hline Total & & 1572 \\
\hline
\end{tabular}

The distribution of specimen is given in table 1 . Among the 1572 specimen screened, 06 specimens were positive for
AFB on smear microscopy. All positive sample showed AFB on centrifuging the sample whereas all of them remained negative on direct smear microscopy.

\section{Discussion}

Extra pulmonary tuberculosis produces a wide spectrum of clinical manifestation which poses a challenge for effective diagnosis. ${ }^{6}$ In general, extrapulmonary TB affects persons with diabetes and HIV, as well as young children $(<15$ years of age) and older adults ( $>65$ years of age). Recent studies have revealed that women and persons who migrate from areas of high TB incidence are at greater risk for extrapulmonary TB. ${ }^{7-9}$ The prevalence of EPTB ranges from 12 to $28.5 \%$ worldwide. In countries like India the prevalence of EPTB ranges from $15-20 \%$ among general population to $50 \%$ among HIV co-infected individuals. ${ }^{5}$ The common forms of EPTB are Lymph node and Lymphatic, Pleural, Urogenital, skeletal and CNS TB. The incidence of different forms of EPTB varies with geographical location. In developed Nations, there is predominance of Lymph node \& Lymphatic TB, whereas in developing Nations, pleural TB is most common followed by Lymphatic TB. ${ }^{10-14}$

The positivity rate of detection of AFB, on smear microscopy, was less than one percentage. The sensitivity of smear microscopy for detection of AFB ranges from 45-60\%. ${ }^{15,16}$ A minimum of 5000 to 10,000 bacilli should be present in one $\mathrm{ml}$ of specimen for it be visualised under routine microscopy. The bacillary load in EPTB, unlike pulmonary TB, is usually not to such extend for it to be visualised in smear microscopy. Hence, the diagnosis of EPTB is based either on culture proven or clinical suspicion with initiation of ATT.

It must be noted in our study that, the specimen processed in our laboratory was irrespective of request for AFB study. The specimens, which were positive for AFB, did not carry any requisition for AFB staining/ culture. Hence, it is prudent to note that, even in unsuspected cases of EPTB a small proportion of case still harboured AFB. However small be the proportion of the cases, it is still significant while considering the epidemiology of tuberculosis. In the absence of suspicion, such undetected cases would be treated as any chronic infection with antibiotics and in many cases with adjunct steroids. This is not only a waste of resource but also increase the patient morbidity \& mortality, cost of patient care and unnecessary health care visits \& hospitalisation. The delayed initiation of specific ATT in such cases is an ideal setting for spread of the infection in the community. In the era of increasing incidence of Multi Drug Resistant (MDR), it may not be justified to provide nonspecific antimicrobial chemotherapy to patients. Furthermore, with large number of patients with HIV co-infection, the disease manifestation may be varied to extent of masking the classical signs and symptoms of Tuberculosis. 


\section{Conclusion}

The findings of the present study, though statistically miniscule, have greater implication at the community level, where controlling tuberculosis by breaking the transmission is of utmost priority. Our findings are based on only by introducing an additional investigation of low sensitivity, i:e smear microscopy. The proportion of cases detected could be higher if Mycobacterial culture or a Rapid molecular method was performed on the specimens. Introduction of a Mycobacterial culture or a rapid Molecular method, like CB NAAT/ GeneExpert, may not be a financially viable option in resource poor countries like India. Nevertheless, it is in these resource poor Nation where the incidence of tuberculosis is high with large proportion EPTB going unnoticed. More evidence needs to be gathered by further studies to prove the utility of introducing an additional investigation as a routine practices.

\section{Limitations of the Study}

Mycobacterial culture could have been performed in a representative sample size. However, the authors did not anticipate such findings and could not make appropriate arrangements for Mycobacterial culture.

\section{Acknowledgement: None \\ Conflict of Interest: None \\ References}

1. Sanjeev H, Karnaker VK, Rai R et al. Rapid Slide Culture: Relevance to the Modern Day Diagnosis of Tuberculosis. Journal of Clinical and Diagnostic Research 2012; 6(3): 378-381.

2. World Health Organization. Global tuberculosis report 2018.WHO/CDS/TB/2018.20. Geneva: The Organization; 2018.

3. Kulchavenya E. Extrapulmonary tuberculosis: are statistical reports accurate. Ther Adv Infect Dis 2014; 2: 61-70.

4. Moore AJ, Evans CAW, Gilman RH et al. MicroscopicObservation Drug Susceptibility Assay for the diagnosis of TB. N Eng J Med 2006; 355: 1539-1550.

5. Velingker A, Lawande D, Dcosta L. Clinico-epidemiological profile of extra pulmonary tuberculosis in Western India. International Journal of Contemporary Medical Research 2018; 5(2): B1-B4.

6. Solovic I, Jonsson J, Korzeniewska-Koseła $\mathrm{M}$ et al. Challenges in diagnosing extrapulmonary tuberculosis in the European Union, 2011. EuroSurveill 2013; 18: 20432.

7. Yang $Z$, Kong $Y$, Wilson $F$ et al. Identification of risk factors for extrapulmonary tuberculosis. ClinDis. 2004;38:199-205. http://dx.doi.org/10.1086/380644.

8. Forssbohm $M$, Zwahlen $M$, Loddenkemper $R$ al. Demographic characteristics of patients with extrapulmonary tuberculosis in Germany. Eur Respir J 2008; 31: 99-105. http://dx.doi. org/10.1183/09031936.00020607.

9. Sreeramareddy $\mathrm{CT}$, Panduru KV, Verma $\mathrm{SC}$ et al. Comparison of pulmonary and extra pulmonary tuberculosis in Nepal: a hospital-based retrospective study. BMC Infect Dis 2008; 8: 8. http://dx.doi. org/10.1186/ 1471-2334-8-8.

10. Arora VK, Gupta R. Trends of extrapulmonary tuberculosis under Revised National Tuberculosis Control Programme: A Study from South Delhi. Indian J Tuberc 2006; 53: 77-83.

11. ZenebeY, Anagaw B, Tesfay W et al. Smear positive extrapulmonary tuberculosis disease at Gondar Hospital, Northwest Ethopia. BMC Research Notes 2013, 6: 21.

12. Fiske $\mathrm{CT}$, Griffin $\mathrm{MR}$, Erin $\mathrm{H}$ et al. Black race, sex and extrapulmonary tuberculosis risk an observational study. BMC Infect Dis 2010; 10: 16.

13. Cailhol J, Decludt B, Che D. Socio demoghraphic factors that contribute to the development of extrapulmonary tuberculosis were identified. I Clin Epidemiol 2005; 58: 1066-1071.

14. Sreeramareddy CT, Panduru KV, Verma SC et al. Comparison of pulmonary and extrapulmonary tuberculosis in Nepal, a hospital based retrospective study. BMC Infect Dis 2008, 8: 8.

15. Parekh KM, Inamdar V, Jog A et al. A comparative study on the diagnosis of pulmonary tuberculosis by using conventional tools and polymerase chain reaction. Ind J Tub 2006; 53: 69-76.

16. Perkins MD, Roscigno G, Zumla A. Progress towards improved tuberculosis diagnostics for developing countries. Lancet 2006; 367: 942-943. 